\title{
Disease Detection in Tomato Plants Using Deep Learning
}

\author{
Marimuthu $\mathrm{S}^{\mathrm{a}, 1}$, Bhuvana $\mathrm{J}^{\mathrm{b}}$ and Mirnalinee $\mathrm{T} \mathrm{T}^{\mathrm{b}}$ \\ ${ }^{\mathrm{a}, \mathrm{b}}$ Dept of CSE, SSN College of Engineering, Chennai, India
}

\begin{abstract}
Agriculture is the backbone of the economy of any country. Productivity of the crops depends on soil quality, proper irrigation and fertilizer, appropriate pesticide. Mostly pesticides were applied without having knowledge about the type of diseases or pests. Type and the quantity of pesticide for any depends on the disease category. If we could identify the appropriate disease, then applying appropriate pesticide will increase the yield of the crop. To address this issue, we propose a method to detect the diseases in tomato plants. We have designed a Convolutional Neural Network architecture that efficiently detects the disease of tomato plant. The system is evaluated with Plant Village benchmark dataset. Results show that our network is detecting the diseases with $90.86 \%$ accuracy. We have identified a suitable variant of Convolutional Neural Network that efficiently detects the disease of tomato plant.
\end{abstract}

Keywords. Disease detection, Tomato Leaves, Convolutional Neural Network, Deep Learning, Agriculture.

\section{Introduction}

Diseases in plants cause considerable production and economic loss in agriculture. Tomato plays main role in Agriculture sector [1]. Basically, tomato has rich nutrition, unique taste and possible health benefits. Tomato can prevent humans from cancer. So, it is essential to increase the productivity of the tomato. Mostly pesticides were applied without having knowledge about the type of diseases or pests. Hence, we need to detect the exact type of disease in order to handle them and to recover the plant from that disease. Today machine learning is playing important role in image detection, face recognition, text detection applications. Several machine learning techniques are generally employed to detect the particular disease of a plant and to suggest proper pesticides for that detected disease. With the evolution of deep learning techniques, Convolutional Neural Network (CNN) [2][3][4][5][6] is found to be one of the important techniques used for image classification method. Detecting the diseases in a particular crop can also be treated as a classification problem. The advantage of using deep learning architecture instead of conventional machine learning is, the automatic extraction of features. Machine learning classifiers on images require hand engineered features to be extracted, wherein one has to determine the nature of features. A deep learning model by its design does not need any human intervention to extract the features, which will be done automatically. In this paper we have designed a deep learning architecture of CNN that classifies 10 different diseases found in the tomato plant. The paper is organized as follows, the section II

Marimuthu S, Department of CSE, SSN College of Engineering, Chennai, India;

E-mail: marimuthu16059@cse.ssn.edu.in. 
discusses about the similar literature available, section III elaborates the proposed architecture for disease classification. The results are discussed in the section IV, where section $\mathrm{V}$ concludes the paper.

\section{Literature Survey}

Diseases will easily destroy the crops, by reducing the yield. If disease detection techniques were to detect the category of disease in advance, the productivity of crops could be improved. The diseases can affect plant leaves, stem but leaves [1][7] are more susceptible which can affect other parts of the plant and hence the productivity. Deep learning approach that makes use of LeNet architecture to classify the diseases in Banana leaves early is reported in [1][7]. LeNet architecture has two stages namely the self-taught feature extraction and classification. This algorithm classifies the leaves in to two classes out of healthy leaves. The disease identified are Black Sigotoka and Black Speckle [8][9]. The disease identification and classification of tomato leaf data set with 200 images has three stages of working [8][10]. Image segmentation has used multi- thresholding, feature extraction used Gray Level Cooccurrence Matrix (GLCM) whereas the classification is done by Support Vector Machine (SVM) [1]. The 70\% of images used for training and $30 \%$ of images are used for testing. The classifier classifies the data set into three classes namely, Bacterial Spot, Early Blight, Spider Mite and healthy leaves with an accuracy of 98.3\%. Potato diseases were identified and classified using image processing and machine learning methods. A data set of 300 images was used to identify three classes namely, Late Blight, Early Blight and healthy leaves. The algorithms used for classification and feature extraction are Support Vector Machine and Gray Level Co-occurrence Matrix respectively [1]. An accuracy of $95 \%$ was obtained by the system for $60 \%-40 \%$ training and testing model.

\section{Methodology}

This section elaborates the architecture of the proposed model to classify and to detect the diseases in tomato leaves.Automatic disease detection in plants is the need of day in order to increase the productivity of the plants. We have designed a deep learning based network to detect the diseases in plants. Our proposed work will use Tomato leaf images which will be given to deep learning network that identifies the type of disease. This work has focused on designing a suitable deep learning network for disease detection by tuning the hyper parameters empirically. The affected tomato plant leaf images have been collected from the Plant Village bench mark dataset for the classification. Totally we have 7166 tomato leaf images available in the dataset. These images are divided into training and testing of $80 \%$ and $20 \%$ each respectively. The proposed classifier is designed to detect 10 different diseases. The diseases that are to be classified along with the healthy leaves of tomato are bacterial spot, early blight, late blight, leaf mold, septoria leaf spot, two spotted spider mite, target spot, mosaic virus, yellow leaf curl virus.

Deep learning Convolutional neural network (DCNN) can be used for the classification of images. The $\mathrm{CNN}$ has three parts such as Convolutional, Pooling and Fully Connected layers. The convolution and pooling layer can act as feature extractors. The early convolutional layers with kernel will extract low level features 
from the leaf images, which will be represented in the form of feature maps or Convolution map. These feature maps are fed to pooling layers to reduce the dimensionality of the intermediate output, thereby reducing the parameters and the computation overhead. This is otherwise referred to as sub sampling. The feature maps are again given to the next set of convolutional layers, where the higher-level features are learnt from accumulating the low-level ones. These extracted features are then given to the fully connected layer for classification. In our classification, the training images are given to the convolutional layer for the feature extraction. This layer has several filters or kernels which are placed over the image and are used to extract the features from the input image. The output of each convolution layer is given to a pooling layer.We have used various filter sizes to identify the best possible architecture to detect the diseases in the tomato plant images. We have built 4 different variants of CNN architecture by varying the filter size. $3 \times 3,5 \times 5,7 \times 7$ and $11 \times 11$ are the various filter sizes used to build the models. All the variants have 3 convolutional layers paired with 3 maxpooling layers. All the convolutional layers are employed with Relu as the activation function. The fully connected layer is implemented through the Dense layer with the number of classes as 10 and softmax as the activation function.

The architecture of the proposed model is given as a model plot in Figure 1. W1 x H1 x D1 be the width, height and depth of the input image. Input image of size 256 $\mathrm{x} 256$ with 3 channels is fed to the first convolutional layer as input, which has 32 filters, each of size $3 \times 3$. The feature map generated by this layer is $\mathrm{W} 2 \times \mathrm{H} 2 \times \mathrm{D} 2$. The size of the feature map is computed as given in Eqs. 1, 2 and 3.

$$
\begin{aligned}
& \mathrm{W} 2=(\mathrm{W} 1-\mathrm{F}+2 \mathrm{P}) / \mathrm{S}+1 \\
& \mathrm{H} 2=(\mathrm{H} 1-\mathrm{F}+2 \mathrm{P}) / \mathrm{S}+1 \\
& \mathrm{D} 2=\mathrm{K}
\end{aligned}
$$

$\mathrm{F}$ is the size of each filter; $\mathrm{P}$ is the padding; $\mathrm{S}$ is the stride and $\mathrm{K}$ being the number of filters. We have not used any padding to retain the same input and output size. Stride is the step size at which the kernel/filter is slide over the receptive area of the input image. With $\mathrm{W} 1=256, \mathrm{H} 1=256, \mathrm{~F}=3, \mathrm{P}=0, \mathrm{~S}=1$, the size of the feature map will be $254 \times 254 \times 32$. The same computation is followed by the consecutive two convolutional layers. Maxpooling layer follows the same convention with pool size 2 x 2 and stride 2.This layer takes 2 x 2 input and determines the maximum of all the four pixels and produces that as the output. The activation function Relu applies the function $f(x)=\max (0, x)$ to all of the values in the input volume. This activation function also helps to handle the vanishing gradient problem, which is the issue where the lower layers of the network train very slowly since the gradient decreases exponentially through the layers. To alleviate the over-fitting problem, the dropout layer drops out a random set of activations in that layer by setting them to zero.

A fully connected layer expects a 1D vector of numbers, hence flattening is applied to simply arranging the $3 \mathrm{D}$ volume of numbers into a $1 \mathrm{D}$ vector. The fully connected layer gets the extracted features from the previous feature extraction part of CNN. The last fully connected layer employs the softmax activation function in order to compute the classes. The output of the last fully connected layer will be a probability that identifies to which the class does an image belongs to. 


\section{Experimental Results and Evaluation}

The training set of 5733 images with 10 different classes of images is given to the CNN for building the model. The trained model is then tested with the test set of 1433 images. Sample leaves from plant village dataset is shown in Figure 2.

We have implemented the variants of the proposed architecture in Python using the deep learning library Keras. The model building and testing are done using Google colab. The model used adam as the optimizer to optimize the error function with learning rate of about 0.001 . The batch size considered while building the model is 32 . The other tunable parameters are listed in Table 1. All the variants are executed for 100 epochs.

All the four variants of the model built follow the same model parameters except the filter size. The model with the filter size $3 \times 3$ convolutional layer has given good accuracy value, when it is compared against the other 3 models. This model gives $90.86 \%$ of accuracy with a loss of about 0.9416 for testing. The testing accuracy and the testing loss for the other three variants of $\mathrm{CNN}$ models are reported in Table 2. The plot corresponding to accuracy and loss for training, and testing are depicted in Figure $1 \& 2$

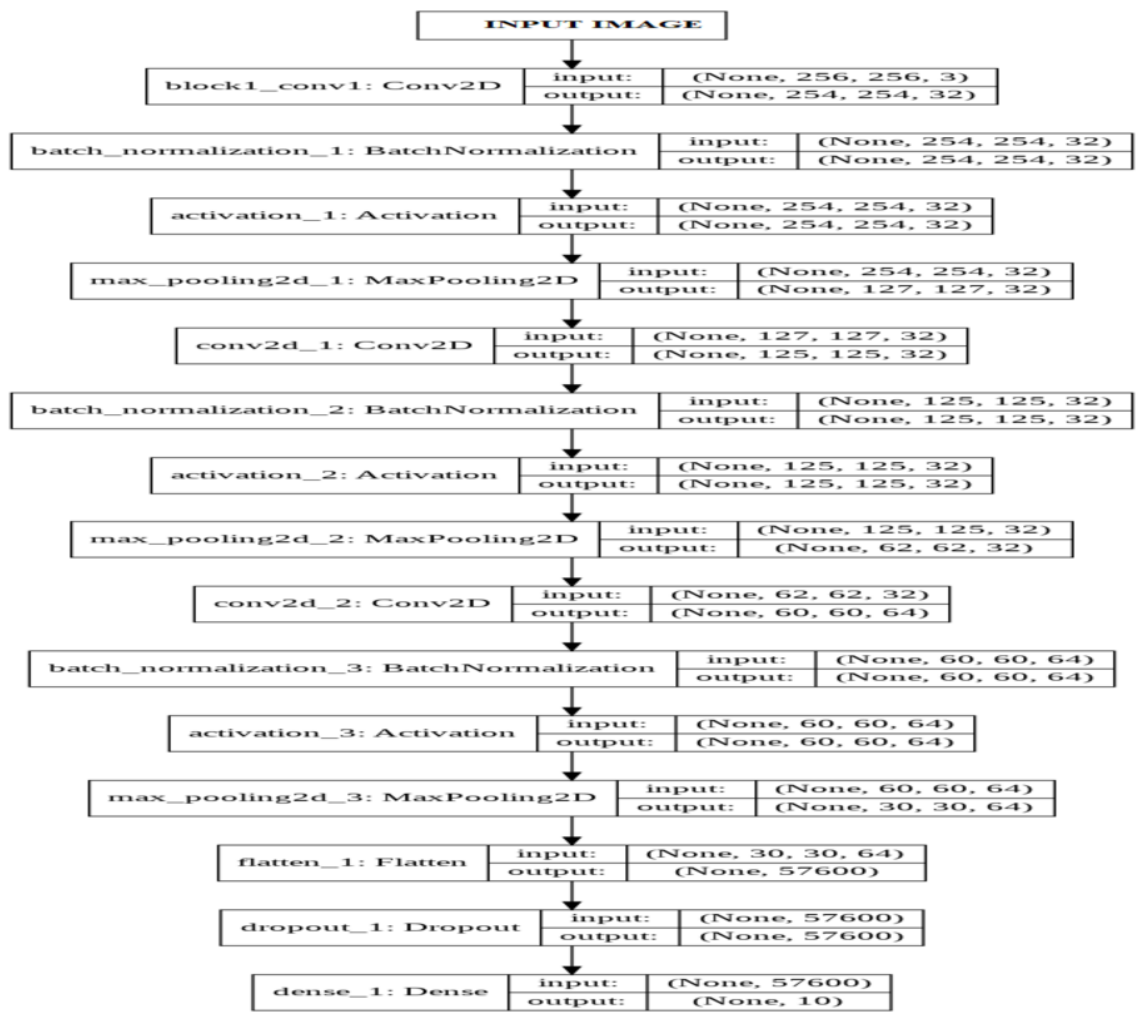

Figure 1. Model plot of CNN variant with $3 \times 3$ filter 

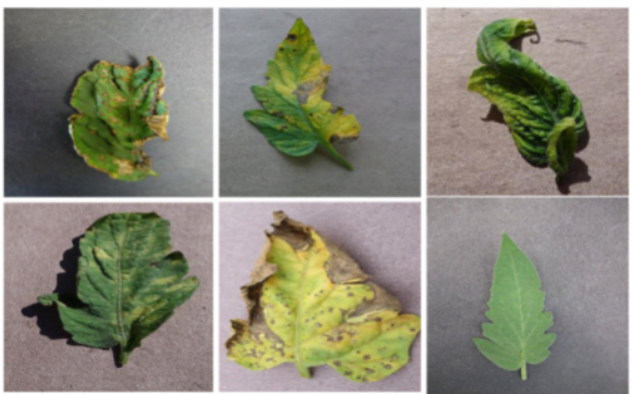

Figure 2. Sample tomato leaves of plant village dataset from bacterial spot, early blight, late blight, septoria leaf spot, yellow leaf curl virus and healthy

TABLE 1. TUNABLE PARAMETERS OF THE PROPOSED ARCHITECTURE

\begin{tabular}{lc}
\hline \multicolumn{1}{c}{ Model parameters } & Value \\
\hline Number of Layers & 12 \\
\hline Batch Size & 32 \\
\hline Learning rate & 0.001 \\
\hline Optimizer & Adam \\
\hline Epochs & 100 \\
\hline Activation functions used & Relu, Softmax \\
\hline Filter sizes & $3 \times 3,5 \times 5,7 \times 7,11 \times 11$ \\
\hline Dropout rate & $50 \%$ \\
\hline
\end{tabular}

Table 2. ACCuracy And Loss Obtained For 4 Variants Of Proposed CnN

\begin{tabular}{llll}
\hline Variant & Filter size & Accuracy & Loss \\
\hline 1 & $3 \times 3$ & $90.86 \%$ & 0.9416 \\
\hline 2 & $5 \times 5$ & $80.80 \%$ & 3.01771 \\
\hline 3 & $7 \times 7$ & $73.97 \%$ & 4.1653 \\
\hline 4 & $11 \times 11$ & $28.54 \%$ & 11.5177 \\
\hline
\end{tabular}


From the obtained results, it is found that the CNN variant with largest filter size $11 \mathrm{x}$ 11 has given poor accuracy and high loss. From this we infer that, for the tomato leaf images of plant village dataset the $11 \times 11$ filter size is not suitable to extract the required features to classify them into appropriate disease classes. We have compared our proposed model with highest accuracy, CNN with $3 \times 3$ filter with the results of SVM in [1]. They have obtained an accuracy of $98.3 \%$ with 10 -fold cross validation. They have used SVM as classifier with image segmentation and feature extraction. In image segmentation, they have used multi-thresholding technique. We have observed that the CNN performance can be further improved by tuning the model parameters and can be checked with a greater number of epochs.

\section{Conclusion and Future Scope}

During the last few years, the performance of convolutional neural networks has been drastically increased compared to traditional hand-craft feature methods of computer vision. In this paper we have proposed a deep learning architecture to identify the diseases in tomato plant. We have experimented with four variants of CNN by changing the filter size. The model with $3 \times 3$ filter has performed well with an accuracy of $90.86 \%$. The advantage of using $\mathrm{CNN}$, being the features are automatically extracted without human intervention. This work has given an insight of working with $\mathrm{CNN}$ on leaf images. The performance can be enhanced further by varying the model parameters. Our proposed system will help in detecting the 10 different diseases in tomato plant leaf images. The architecture used in this work can also be used to detect diseases in other plants as well.

\section{References}

[1] Ud Din Z, Adnan SM, Ahmad RW, Aziz S, Ismail W, Iqbal J. Classification of Tomato Plants Leaf Diseases Using Image Segmentation and SVM. Technical Journal. 2018 Aug 30;23(02):81-8.

[2] Mohanty SP, Hughes DP, Salathé M. Using deep learning for image-based plant disease detection. Frontiers in plant science. 2016 Sep 22;7:1419.

[3] Chaerani R, Voorrips RE. Tomato early blight (Alternaria solani): the pathogen, genetics, and breeding for resistance. Journal of general plant pathology. 2006 Dec 1;72(6):335-47.

[4] Tang Y, Wu X. Text-independent writer identification via CNN features and joint Bayesian. In2016 15th International Conference on Frontiers in Handwriting Recognition (ICFHR) 2016 Oct 23 (pp. 566571). IEEE.

[5] Krizhevsky A, Sutskever I, Hinton GE. Imagenet classification with deep convolutional neural networks. In Advances in neural information processing systems 2012 (pp. 1097-1105).

[6] Yakkundimath R, Saunshi G, Kamatar V. Plant Disease Detection using IoT. Global Journal of Computer Science and Technology. 2018 Sesp.

[7] Mokhtar U, El Bendary N, Hassenian AE, Emary E, Mahmoud MA, Hefny H, Tolba MF. SVM-based detection of tomato leaves diseases. InIntelligent Systems' 20142015 (pp. 641-652). Springer, Cham.

[8] Amara J, Bouaziz B, Algergawy A. A deep learning-based approach for banana leaf diseases classification. Datenbanksystemefür Business, Technologie und Web (BTW 2017)-Workshopband. 2017.

[9] Wang G, Sun Y, Wang J. Automatic image-based plant disease severity estimation using deep learning. Computational intelligence and neuroscience. 2017;2017.

[10] Barbedo JG. Digital image processing techniques for detecting, quantifying and classifying plant diseases. SpringerPlus. 2013 Dec 1;2(1):660. 